\title{
Handover Performance of HVAC Duct Based Indoor Wireless Networks
}

\author{
A. E. Xhafa, P. Sonthikorn ${ }^{\dagger}$, and O. K. Tonguz ${ }^{\dagger}$ \\ Texas Instruments Inc., Dallas, TX 75243, USA \\ Email: axhafa@ti.com \\ † Carnegie Mellon University, Department of Electrical and Computer Engineering \\ Pittsburgh, PA 15213-3890, USA \\ Email: \{psonthik,tonguz\}@ece.cmu.edu
}

\begin{abstract}
In this paper, we present a performance analysis for different implementations of handover in indoor wireless networks (IWN) that use heating, ventilation, and air conditioning (HVAC) ducts as communication channels. If FDMA/TDMA technology is used, our results show that the handover performance, i.e., new call blocking and handover dropping probabilities, of an IWN that uses HVAC ducts are up to 6.6 times better for practical scenarios compared to those of traditional IWN (i.e., IWN that do not use HVAC ducts). Our results also indicate that for scenarios under investigation, using a single access point to serve one floor and its staircase region achieves the best handover performance for IWN that use HVAC ducts.

The solution to the handover problem in HVAC-IWN is tightly coupled with the coverage, capacity, and load balancing issues in indoor wireless networks. We show, for the first time, that the proposed solution to the handover problem in HVAC-IWN results in an increase in capacity per coverage area, an increase in the radius of coverage for an access point, and can be used to achieve load balancing in WLANs that use IEEE 802.11 technology.
\end{abstract}

\section{INTRODUCTION}

To ensure service continuity, seamless handover in future wireless networks is one of the important system issues that needs to be addressed. Numerous studies have been done to investigate the handover issue in indoor wireless networks (IWN) [1]-[3]. These studies were done in traditional IWN (T-IWN), where the radio frequency (RF) signal is transmitted/received via a network of transmitters/receivers and antennas placed throughout the interior of a building [4].

An alternative approach to transmitt/receive the RF signal is to use heating, ventilation, and airconditioning (HVAC) ducts [5]. To deal with the handover issue in the IWN that use HVAC ducts (HVAC-IWN), a new system architecture design was proposed in [1]. Figure 1 illustrates this architecture. The IWN that uses the HVAC ducts are connected to the outside IP-network via the access point controller (APC). The APC consists of End User Mobile Switch and access points [1]. The APC serves the whole building and is connected to one or more antennas in the duct. Each antenna acts as a remote antenna (RA) for a particular floor, or for a group of floors. Figure 1 shows an APC connected to an RA that serves three floors in a building. In this model, each receiving antenna (passive reradiators) in a room/office is placed in the HVAC duct that is located in its corresponding room/office. The RF signal sent from the RA propagates through the ducts and is received by the receiving antennas placed at each room/office. It was shown in [1] that using the aforementioned system architecture, intra-floor handovers can be eliminated while inter-floor handovers can be reduced drastically. However, the handover performance analysis, i.e., new call blocking and handover dropping probabilities, data rate, etc., in the HVACIWN, to date, has not been reported.

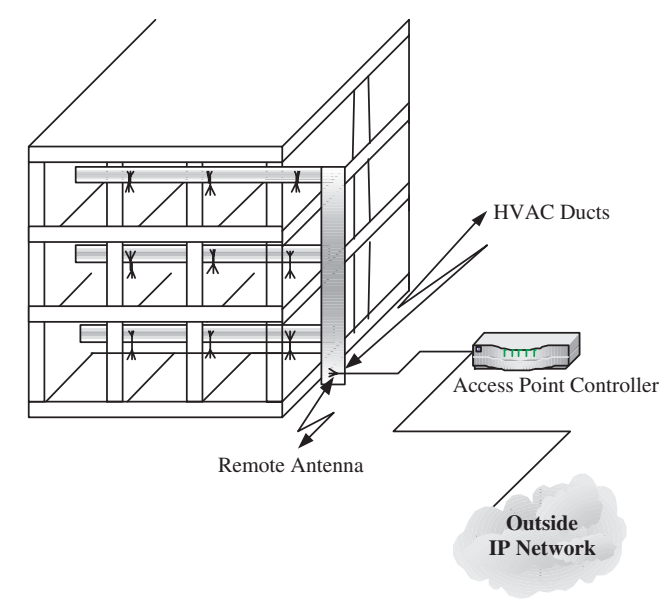

Fig. 1. An HVAC system example.

In this paper, we present a performance analysis of handover in HVAC-IWN and discuss how different implementations of handover can improve network performance in terms of key performance metrics. Our results show that for HVAC-IWN that use FDMA/TDMA technology, the handover performance, i.e., new call blocking and handover dropping probabilities, is up to 6.6 times better for practical scenarios compared to those of T-IWN. Our results also show that for scenarios under investigation, using a single AP to serve one floor and its staircase region is the optimum solution for HVAC-IWN. Furthermore, we show, for the first time, that the proposed solution to the handover problem in HVAC-IWN results in an increase in capacity per coverage area, an increase in the radius of coverage for an access point, and can be used to achieve load balancing in WLANs that use IEEE 802.11 technology.

\section{BACKGROUND AND MOTIVATION}

An alternative approach to providing indoor wireless communications in buildings is to propagate the RF signals through the HVAC ducts. Concepts and preliminary work on this subject have been reported in [5]. 


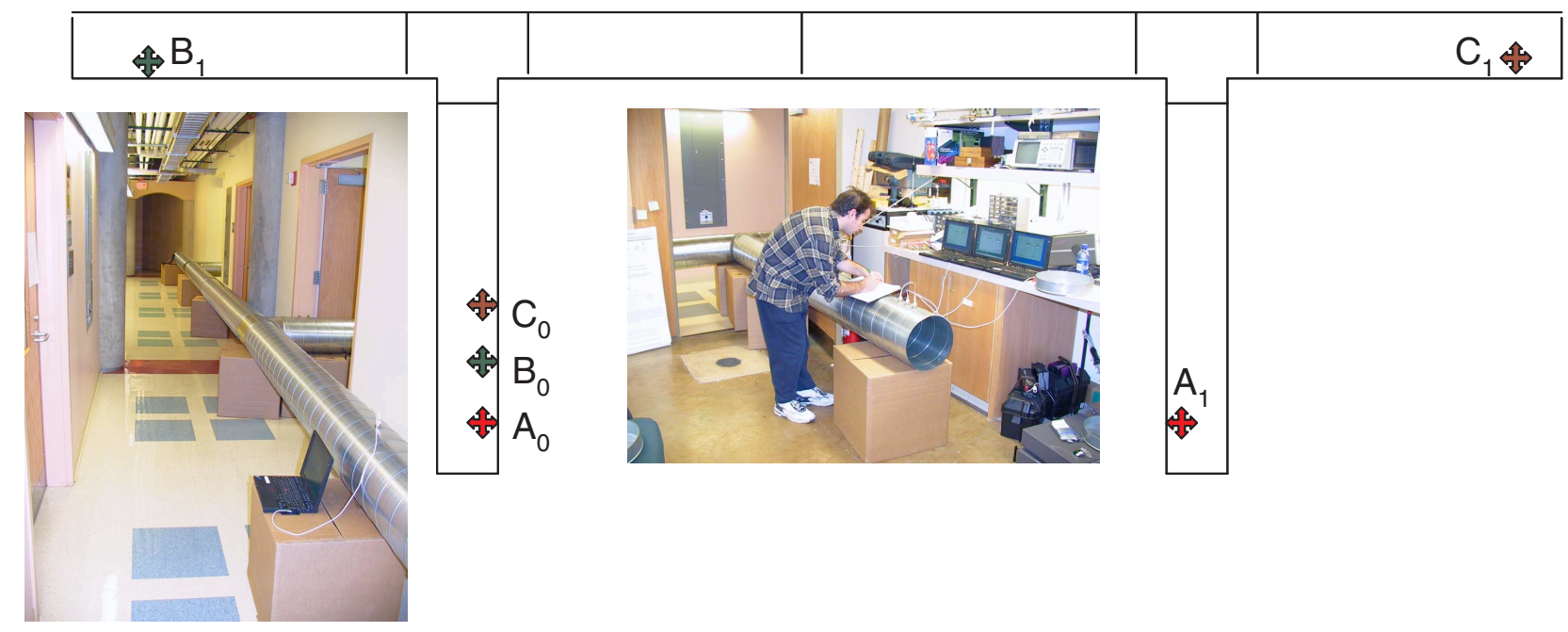

Fig. 2. IEEE $802.11 \mathrm{~b}$ experiment.

Intra-floor handovers can be eliminated if the whole floor of a building is covered by a single access point (AP). This is possible due to the low attenuation of the RF signals in the HVAC ducts [7]. To illustrate the concept of using a single AP to cover the whole floor region, an experiment was done on the second floor of Roberts Hall on Carnegie Mellon University's campus. In the experiment, a test duct network that included tee and straight sections was configured (see Figure 2). The duct components have a diameter of $0.3 \mathrm{~m}$ and are made of galvanized steel with conductivity $\sigma=10^{6} \mathrm{~S} / \mathrm{m}$. The ends of the ducts were left open. The built-in antennas in the Lucent wireless cards were removed and instead, monopole probe antennas of length $3.1 \mathrm{~cm}$ were connected to the Lucent wireless cards via short cables. These monopole probe antennas were then mounted in the ducts and were used for communication between three pairs of laptops. The laptops were using $802.11 \mathrm{~b}$ technology at $2.45 \mathrm{GHz}$ ISM band and operated simultaneously. The laptops were placed at locations $A_{0}, B_{0}, C_{0}, A_{1}, B_{1}$, and $C_{1}$ as shown in Figure 2. Note that laptops placed at locations with the same letter were using the same frequency channel that is non-overlapping with the other frequency channels used by the other laptops. For example, the laptops at locations $A_{0}$ and $A_{1}$, which were 12 $\mathrm{m}$ apart in the duct network, were using frequency channel 1 ; the laptops at locations $B_{0}$ and $B_{1}$, which were $6 \mathrm{~m}$ apart in the duct network, were using frequency channel 6; and the laptops at locations $C_{0}$ and $C_{1}$, which were $12 \mathrm{~m}$ apart in the duct network, were operating at frequency channel 11. These are the only three non-overlapping channels in 2.45 GHz ISM band. The total data rate that was measured was $28 \mathrm{Mbps}$, which is $5 \mathrm{Mbps}$ lower than the total $33 \mathrm{Mbps}$ that all three channels can provide. The reduction in the data rate can be explained with the small overlaps that exist between frequency channels 1,6 , and 11 . The same data rate, $28 \mathrm{Mbps}$, was measured even when we switched the locations between the laptops placed at points $A_{1}, B_{1}$, and $C_{1}$. Thus, one can conclude that using co-located APs (or super AP), where three APs that operate at non-overlapping channels are placed at the same location in the HVAC network, no handover occurs when a mobile user moves from location $A_{1}$ to $B_{1}$ or $C_{1}$ in the network. Therefore, no handover occurs when a mobile user moves on the floor.

While the concept of the HVAC-IWN system architecture has been described in [1], the main objective of this paper is to investigate and quantify the handover performance of HVAC-IWN. Furthermore, we show, for the first time, that the proposed solution to the handover problem in HVAC-IWN results in an increase in capacity per coverage area, an increase in the radius of coverage for an access point, and can be used to achieve load balancing in the WLAN that use IEEE 802.11 technology.

Next we present an analysis for the handover performance of the HVAC-IWN network.

\section{ANALYSIS OF HVAC HANDOVER}

The first part of the analysis deals with the handover performance of the proposed approach in indoor wireless networks that use FDMA/TDMA technology, while the second part of the analysis deals with the handover performance analysis of WLANs that use IEEE 802.11 technology.

\section{A. Indoor Wireless Networks that use FDMA/TDMA Technol- ogy}

1) Traditional Indoor Wireless Networks: We assume that the floor region consists of $N$ cells and that new calls in a given cell are generated at rate $\lambda_{n}$ and follow a Poisson process. We also assume that handover calls follow a Poisson process, however, with rate $\lambda_{h}$. Therefore, the total traffic in a cell is $\lambda=\lambda_{n}+\lambda_{h}$. In a realistic scenario, handover traffic for different cells is different; e.g., cells that are located on the edge of a floor plan have less handover traffic (less than $\lambda_{h}$ ), while the staircase region cells have higher handover traffic (higher than $\lambda_{h}$ ). For the sake of simplicity, we assume that handover traffic in each cell is the same. Furthermore, we assume that the channel holding time for the new and handover calls follows an exponential distribution with mean $1 / \mu_{H}$. Given that a cell has $C$ channels, the handover performance of the cell can be calculated via an $M / M / C / C$ queuing model. Queuing of handover calls that do not find free channels is not considered since the overlapping area between neighboring cells is almost non-existent in IWN. Blocking of new and handover calls occur if all the channels in the cell are busy. Therefore, the new call and handover call blocking 
probabilities are given as (Erlang B formula [8]):

$$
P_{B}=P_{H}=P_{C}=\frac{\frac{1}{C !}\left(\frac{\lambda}{\mu_{H}}\right)^{C}}{\sum_{i=0}^{C} \frac{1}{i !}\left(\frac{\lambda}{\mu_{H}}\right)^{i}}
$$

Assuming that the traffic statistics and the number of channels is the same for all the cells on the floor (floor region and staircase region cells), the average new and handover blocking probabilities on the floor are also given by Eqn.(1) in T-IWN. Next, we consider different scenarios in HVAC-IWN.

2) Indoor Wireless Networks that use HVAC: Scenario A: In this scenario, the number of channels used in the staircase region (cell) and in each of the cells in the floor region is the same and equal to $C$. Furthermore, we assume that in the case of HVAC-IWN, each AP serves only one floor region (this is possible due to the low attenuation of the RF signals in the HVAC ducts [7]), and another AP serves the staircase region (cell). The new call generation rate and the handover rate in the staircase region are assumed to be $\lambda_{n}$ and $\lambda_{h}$, respectively. The traffic for the other cell, which is the floor region cell, however, is different. The new call generation rate is $N \lambda_{n}$, since the floor region includes $N$ cells. Assuming that there is an equilibrium flow between the staircase region and the floor region and since the staircase region cell is the only neighboring cell to the floor region cell, the handover traffic to the floor region cell is $\lambda_{h}$. Furthermore, we assume that the number of channels for the staircase region cell remains the same, $C$, while for the floor region cell, this number is $C_{n}=$ $N C$, since $N$ cells make the floor region cell. The new call and handover call blocking probabilities for the staircase region cell can be calculated from Eqn.(1), while for the floor region cell can be calculated from the $M / M / C_{n} / C_{n}$ queuing model. Hence, the new call and handover call blocking probabilities for the floor-region cell, $P_{B}^{F R}$ and $P_{H}^{F R}$, respectively, are given as:

$$
P_{B}^{F R}=P_{H}^{F R}=\frac{\frac{1}{C_{n} !}\left(\frac{N \lambda_{n}+\lambda_{h}}{\mu_{H}}\right)^{C_{n}}}{\sum_{i=0}^{C_{n}} \frac{1}{i !}\left(\frac{N \lambda_{n}+\lambda_{h}}{\mu_{H}}\right)^{i}}
$$

Therefore, the average new call and handover call blocking probabilities on the floor (i.e., floor and staircase regions) are given as:

$$
\begin{aligned}
P_{B}^{F}=P_{H}^{F}= & \frac{N \lambda_{n}+\lambda_{h}}{(N+1) \lambda_{n}+2 \lambda_{h}} P_{B}^{F R} \\
& +\frac{\lambda_{n}+\lambda_{h}}{(N+1) \lambda_{n}+2 \lambda_{h}} P_{B}
\end{aligned}
$$

3) Indoor Wireless Networks that use HVAC: Scenario B: In this scenario, we assume that each AP serves $k$ floor regions and $k$ staircase regions. The benefit of such an architecture is that there is no handover between the $k$ floor regions and $k$ staircase regions, thus, it completely eliminates intra-floor handovers and it greatly reduces inter-floor handovers. The total traffic supported by a single AP is given as:

$$
\lambda_{t o t}=k(N+1) \lambda_{n}+\lambda_{h}
$$

where the handover traffic accounts for the handover requests arriving from above/below floors. The total number of channels available to the $\mathrm{AP}$ is $C_{n+1}=k(N+1) C$, where the factor $(N+1)$ is due to the fact that the whole floor consists of the floor region (that consists of $N$ cells) and the staircase region (that consists of 1 cell). Hence, the new call and handover dropping probabilities are given as:

$$
P_{B}^{F}=P_{H}^{F}=\frac{\frac{1}{C_{n+1} !}\left(\frac{\lambda_{t o t}}{\mu_{H}}\right)^{C_{n+1}}}{\sum_{i=0}^{C_{n+1}} \frac{1}{i !}\left(\frac{\lambda_{t o t}}{\mu_{H}}\right)^{i}}
$$

\section{B. 802.11 Indoor WLAN}

Since throughput and delay are the main performance metrics of these networks, it is reasonable to expect that the main concern for handoffs will be data rate or throughput and latency. In this analysis, we investigate the impact of the system architecture on the data rate of the mobile users in a multi-story building.

802.11 WLANs operate in $2.45 \mathrm{GHz}$ ISM band. There are three non-overlapping channel frequencies, which can be used to provide internet access on a given floor. Therefore, in TIWN, a given floor is partitioned in three cells, each using one of the non-overlapping channels. In the case of HVACIWN, we consider the case when a single AP, that transmits and receives RF signals in all three non-overlapping channels, is used to serve the floor region and the staircase region. Therefore, the handovers are confined to inter-floor handovers only. Thus, in the case of T-IWN the traffic rates in cell 1 and 3 are $\lambda_{n}+\lambda_{h}$, while in cell 2 is $\lambda_{n}+4 \lambda_{h}$. We have assumed that cell 1 and 3 have only one neighboring cell, cell 2; while cell 2 has cell 1 , cell 3 , and the cells in the floors above and below the floor under consideration. This is the reason why the handover rates for cell 1 and 3 is only $\lambda_{h}$, while for cell 2 is $4 \lambda_{h}$. In HVAC-IWN the traffic rate in the "supercell" is $3 \lambda_{n}+2 \lambda_{h}$, where $\lambda_{h}$ accounts for the handover traffic arriving from the floors above and below the floor under consideration.

To calculate the data rate provided to the mobile terminals in both approaches, T-IWN and HVAC-IWN, let us assume that the data rate provided in each of the non-overlapping channels is $D$. The average number of connections being served by a single AP (that operates in one of the non-overlapping channels) is given as:

$$
N_{M T}=\sum_{n=0}^{\infty} n p_{n}
$$

where $p_{n}$ denotes the probability that there are $n$ users connected to the AP and is given as:

$$
p_{n}=\rho^{n}(1-\rho)
$$

and $\rho=\lambda / \mu_{H}$, where $1 / \mu_{H}$ is the time duration for a connection and $\lambda$ is the traffic load. Note that Eqn.(7) is valid for WLAN that use spread spectrum technology and under the condition that the arrival rate (i.e., connection rate) is Poisson. Also note that Eqn.(7) is independent on the distribution of the service time duration [9], [10]. Thus, the data rate provided to the mobile terminals connected to an AP in T-IWN is given as:

$$
D_{M T}=\max \left\{D_{\min }, \min \left(D / N_{M T}, D_{\max }\right)\right\}
$$

where $D_{\max }$ denotes the maximum data rate that can be provided in a single channel, and $D_{\min }$ is the minimum data rate necessary to keep the connection alive.

In the case of HVAC-IWN, the analysis is similar; however, the traffic load in this case is different. If we assume that each of the channels carries the same traffic, then the arrival rate per AP is given as:

$$
\lambda_{a p}=\lambda_{n}+\frac{1}{N} \lambda_{h}
$$


where $N$ denotes the number of access points collocated on a given floor. The normalized load supported by each AP is given as:

$$
\rho_{a p}=\frac{\lambda_{a p}}{\mu_{H a}}
$$

where $1 / \mu_{H a}$ is the time duration of a connection. The data rate provided to mobile terminals in HVAC-IWN can be found using the same approach as in the case of T-IWN. The benefit of using HVAC-IWN is not an increase in total data rate, rather, it is the increase in coverage, capacity (per area), and load balancing.

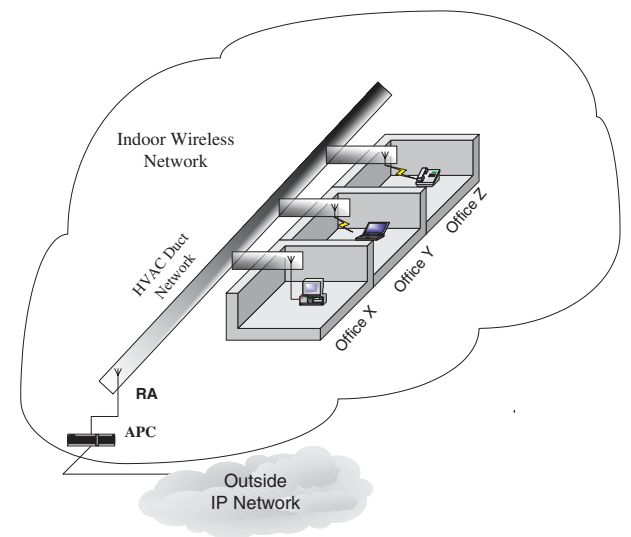

Fig. 3. Indoor wireless network using HVAC ducts in a floor.

To illustrate the increase in capacity, consider the scenario shown in Figure 3. If one uses T-IWN, and assuming that the three offices represent the whole floor, then three APs are needed to provide wireless communications for each of the offices: Office X, Office Y, and Office Z. Thus, the data rate at each of these offices is equal to the data rate supported by a single AP. For example, if IEEE 802.11g technology is used, the data rate provided to each office is $54 \mathrm{Mbps}$. To increase the capacity, one could use three APs (which operate in nonoverlapping channels in $2.4-2.5 \mathrm{GHz}$ band) in each office. Therefore, the data rate provided to each office is three times the data rate provided by each $\mathrm{AP}$, hence $162 \mathrm{Mbps}$. However, this approach results in an increase in the interference level between APs that use the same channel but cover different offices. In addition, the number of APs used in this case is nine, which is substantial. Consider the following approach. One can achieve this by co-locating all three non-overlapping channels at one access point and use three RAs to transmit the RF signals from each of the three non-overlapping channels. Thus, instead of a single RA in Figure 3, there would be three RAs. Each office is now being served by all three nonoverlapping channels; hence, the data rate provided to each of the offices is $162 \mathrm{Mbps}$. Thus, we have increased the capacity in Mbps/area compared to T-IWN without introducing any interference between APs.

Next, consider the following scenario: assume that Office $\mathrm{X}$ is a conference room and during certain time of the day, the amount of data rate required for this office is much higher than the other ones. This could be the scenario if Office $\mathrm{X}$ is used for daily meetings and every participant in this meeting brings his/her laptop. At the same time, other offices, Office Y and Office $\mathrm{Z}$, during these times of the day (i.e., when there is a meeting in Office $X$ ) could require less data rate, since most of the people working in these offices are attending the meeting. Since all three APs are co-located and can provide coverage for the whole floor, load balancing in this case implies that more data rate is accommodated in Office $X$ and less data rate is accommodated in Office $\mathrm{Y}$ and in Office $\mathrm{Z}$. In fact, nothing needs to be done to provide load balancing in this case, since the co-location of APs automatically provides load balancing on the floor.

Next, we present numerical results on the performance of HVAC-IWN.

\section{RESULTS AND DiscusSiONS}

In this section we present numerical results obtained for HVAC-IWN, compare them to the results obtained for the TIWN, and discuss their implications.

\section{A. Indoor Wireless Networks that use FDMA/TDMA Technol- ogy}

Figure 4 shows the average new call and handover blocking probabilities in Scenario A versus the number of cells on the floor with different number of channels per cell. Note that the plots should not be continuous, however, using the continous plots make the trends clear. The same argument is applied to the rest of the plots presented in this paper. We assume that the normalized load in a single cell case is 0.6 Erlangs (i.e., $\left.\left(\lambda_{n}+\lambda_{h}\right) / \mu_{H}\right)$, while the probability that a call in progress will experience a handover is 0.5 . It is clear that as the number of channels per cell increases, the improvement increases. For example, for $C=3$ and $N=10, P_{B}^{F} \approx 0.03$, while for $C=2$ and $N=10, P_{B}^{F} \approx 0.04$. Note that as $N$ increases, the performance of HVAC-IWN improves. Also, note that $N=1$ yields the new call and handover call blocking probabilities for the T-IWN and the handover performance of T-IWN does not change as $N$ increases. HVAC-IWN on the other hand, yields better handover performance as $N$ increases. For example, for $N=10$ and $C=3$, the handover performance of HVAC-IWN is 0.03 , while for T-IWN is 0.2. This implies that HVAC-IWN improves the handover performance by 6.6 times.

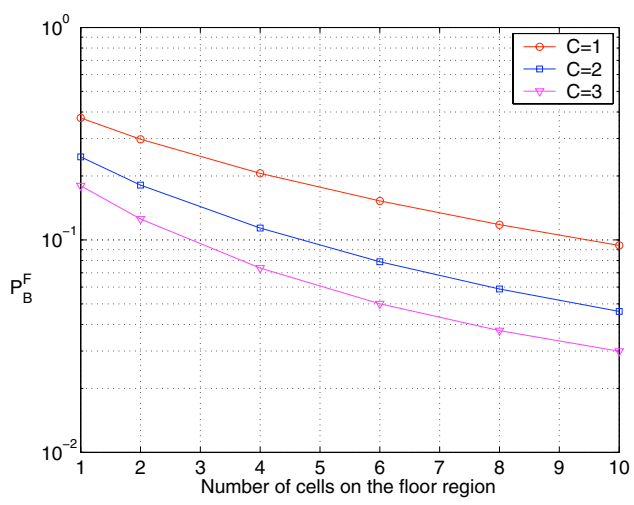

Fig. 4. Average new call and handover call blocking probabilities versus the number of cells for different number of channels per cell.

The handover performance of Scenario $B$ is shown in Figure 5. In this case, the traffic load for a single cell is assumed to be 0.6 Erlangs, the number of channels per cell is 2 , and the number of staircase regions served by the same AP are taken to be 1,2 , and 3 . The plots show the average new call and handover call blocking probabilities versus different number of cells in the floor region and for different values of $k$. One can observe that as the number of cells in the floor region increases, the performance of HVAC-IWN improves. However, as the number of floors and staircase regions served by the same AP increases, the performance of HVAC-IWN degrades. 


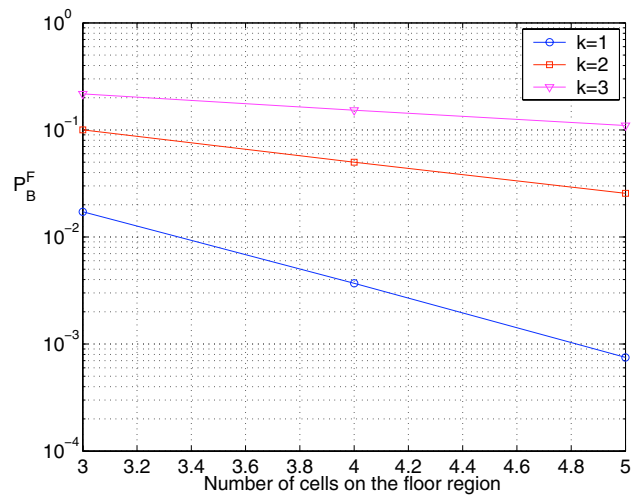

Fig. 5. The performance versus the number of cells and for different values of $k$ in Scenario $B$.

For example, if the number of cells in the floor region is 2 , for $k=1, P_{B}^{F}$ has a value of 0.003 , while for $k=2$, its value is 0.02 , which implies a deterioration by approximately 7 folds. The reason for this is that as the number of floors (i.e., the floors and their respective staircase regions) served by the same AP increases, the traffic load served by this particular AP increases, while the number of channels remains the same. Hence, although using a single AP to serve more than one floor reduces the inter-floor handovers, it results in an increase in new call and handover call blocking probabilities.

Comparing the handover performance of the three scenarios under investigation, it is clear that:

- Scenario $B$ achieves the best performance if a single AP is used to serve a single floor and its corresponding staircase region.

- When $k=3$ in Scenario $B$, its handover performance becomes comparable to that of Scenario A.

- Also note that the number of AP required to serve a multistory building in Scenario $A$ is higher than that used in Scenario B. However, if one considers the handover performance in terms of new call and handover call blocking probabilities only, the optimum solution is to use a single AP to serve the floor and the staircase region.

Regardless of the approach/scenario considered, the improvement in the handover performance of HVAC-IWN compared to the T-IWN is up to 6.6 times better when $N \geq 10$, which is substantial. Note that in selecting the optimum solution that yields the lowest new call and handover blocking probabilities, nothing has been said about the data rate provided to the users. Also, we assume that the handover rate in each cell is $\lambda_{h}$, while in practical scenarios, this might not be the case. Further research is needed to find the optimum solution when the data rate provided to the mobile users is also taken into consideration and when the traffic is not uniform.

\section{B. 802.11 Indoor WLAN}

We investigate the impact of using HVAC technology on the data rate provided to the mobile terminals. We assume that the data rate that can be supported in a single channel is $54 \mathrm{Mbps}$ (IEEE 802.11g), the minimum data rate is $1 \mathrm{Mbps}$, and the probability that a call in progress will experience handover is 0.2 . The data rate provided to the mobile terminals is plotted versus the normalized load. It is clear from Figure 6 that HVAC-IWN provides better performance than T-IWN, because HVAC-IWN reduces the offered load per frequency channel. Furthermore, HVAC-IWN provide higher coverage

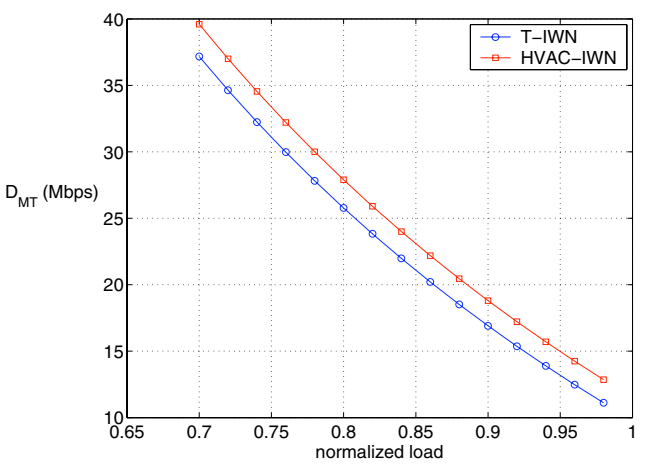

Fig. 6. Data rate versus traffic load for T-IWN and HVAC-IWN.

for the access points, higher data rate per area, and provides load balancing in the indoor wireless networks.

\section{CONCLUSIONS}

In this paper, we presented a handover performance analysis of HVAC-IWN. Our results show that the handover performance of HVAC-IWN is up to 6.6 times better for practical scenarios compared to that of T-IWN. We have also shown that for the scenarios under investigation, the optimum solution is to use a single AP to cover one floor and its staircase region. We also showed, for the first time, that the proposed solution to the handover problem in HVAC-IWN results in an increase in capacity per coverage area, an increase in the radius of coverage for an access point, and can be used to achieve load balancing in the indoor WLANs that use IEEE 802.11 technology.

\section{REFERENCES}

[1] A. E. Xhafa, P. Sonthikorn, O. K. Tonguz, P. V. Nikitin, A. G. Cepni, D. D. Stancil, B. Henty, and D. Brodtkorb, "Seamless handover in buildings using HVAC ducts: a new system architecture", IEEE Global Telecommunications Conference (Globecom'03), vol. 6, pp. 3093-3097, 2003.

[2] G. K. Wolterin, "Handoff, a necessary feature in wireless office systems", 1st International Conference on Universal Personal Communications, 1992. ICUPC '92 Proceedings., pp. 13.06/1 -13.06/6, 29 Sept.Oct. 1, 1992.

[3] T. S. Kim, M. Y. Chung, and D. K. Sung, "Mobility modelling and traffic analysis in three-dimensional indoor environments", IEEE Transactions on Vehicular Technology, Vol. 47, no. 2, pp. 546-557, May 1998.

[4] D. Molkdar, "Review on radio propagation into and within buildings", IEE Proc., vol. 138, no. 1, pp. 61-73, Feb. 1991.

[5] D. D. Stancil, O. K. Tonguz, A. Xhafa, A. Cepni, P. Nikitin, and D. Brodtkorb, "High-speed Internet access via HVAC ducts: A new approach", in Proc. of IEEE Global Telecomm. Conf. (GLOBECOM'01), vol. 6, pp. 3604-3607, San Antonio, Texas, Nov. 2001.

[6] A. Xhafa, O. K. Tonguz, A. Cepni, D. D. Stancil, P. Nikitin, and D. Brodtkorb, "Theoretical Estimates of HVAC Duct Channel Capacity for High-speed Internet Access", Proceedings of IEEE International Conference on Communications (ICC 2002), Vol. 2, pp. 936-939, New York, April 28-May 2, 2002.

[7] O. K. Tonguz, D. D. Stancil, A. E. Xhafa, A. G. Cepni, P. V. Nikitin, and D. Brodtkorb, "An empirical path loss model for HVAC duct systems", in Proc. IEEE Global Telecommunications Conference (GLOBECOM'02), vol. 2, pp. 1850-1854, Taipei, Taiwan, 17-21 Nov. 2002.

[8] D. Gross and C. M. Harris, Fundamentals of queuing theory, 2nd edition, John-Wiley, 1997

[9] A. E. Xhafa, "Analysis, Design, and Implementation of Handover Priority Schemes in Wireless Networks", Ph.D. Thesis, Advisor: Prof. O. K. Tonguz, Carnegie Mellon University, Pittsburgh, PA, USA, October 2003.

[10] S. Ross, Stochastic Processes., Wiley, John \& Sons, Inc., 2nd Edition, 1996. 